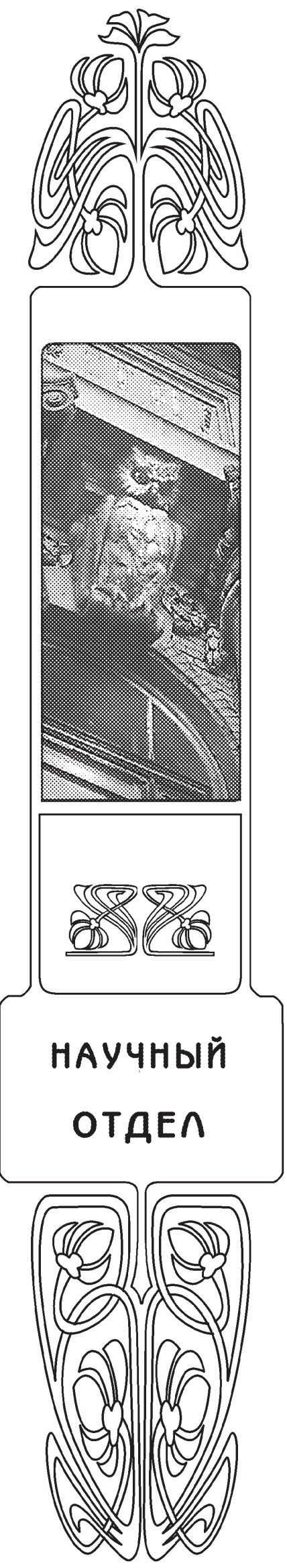

\title{
MATEMATИKA
}

\section{On Semigroups of Relations with the Operation of Left and Right Rectangular Products}

\author{
D. A. Bredikhin
}

Dmitry A. Bredikhin, https://orcid.org/0000-0003-3600-1294, Yuri Gagarin State Technical University of Saratov, 77 Politechnicheskaya St., Saratov 410054, Russia, bredikhin@mail.ru

A set of binary relations closed with respect to some collection of operations on relations forms an algebra called an algebra of relations. The class of all algebras (partially ordered algebras) isomorphic to algebras (partially ordered by set-theoretic inclusion $\subseteq$ algebras) of relations with operations from $\Omega$ is denoted by $\mathrm{R}\{\Omega\}$ $(R\{\Omega, \subseteq\})$. An operation on relations is called primitive-positive if it can be defined by a formula of the first-order predicate calculus containing only existential quantifiers and conjunctions in its prenex normal form. We consider algebras of relations with associative primitive-positive operations $*$ and $\star$, defined by the following formulas $\rho * \sigma=\{(u, v):(\exists s, t, w)(u, s) \in \rho \wedge(t, w) \in \sigma\}$ and $\rho \star \sigma=\{(u, v):(\exists s, t, w)(s, t) \in \rho \wedge(w, v) \in \sigma\}$ respectively. The axiom systems for the classes $\mathrm{R}\{*\}, \mathrm{R}\{*, \subseteq\}, \mathrm{R}\{\star\}, \mathrm{R}\{\star, \subseteq\}$, and bases of quasi-identities and identities for quasi-varieties and varieties generated by these classes are found.

Keywords: algebra of relations, primitive positive operation, identity, variety, quasi-identity, quasi-variety, semigroup, partially ordered semigroup.

Received: 11.06.2019 / Accepted: 28.06.2019/

Published: 31.08.2020

This is an open access article distributed under the terms of Creative Commons Attribution License (CC-BY 4.0)

DOI: https://doi.org/10.18500/1816-9791-2020-20-3-280-289

\section{INTRODUCTION}

Let $\operatorname{Rel}(U)$ be the set of all binary relations on a base set $U$. A set of binary relations $\Phi \subseteq \operatorname{Rel}(U)$ closed with respect to some collection $\Omega$ of operations on relations forms an algebra $(\Phi, \Omega)$ called an algebra of relations. Theory of algebras of relations is an essential part of modern algebraic logic and has numerous applications in theory of semigroups [1]. 
Operations on relations are usually determined using first-order predicate calculus formulas. Such operations are called logical. It is known that classes of algebras of relations with logical operations are axiomatizable [1]. Logical operations can be classified by the type of formulas defining them. An operation on relations is called primitive-positive [2] (in other terminology - Diophantine operations [3,4], generalized superpositions [5]) if it can be defined by a formula of the first-order predicate calculus containing only existential quantifiers and conjunctions in its prenex normal form.

Note that the set-theoretical inclusion $\subseteq$ is compatible with all primitive-positive operations. Thus, any algebra of relations with primitive-positive operations $(\Phi, \Omega)$ can be considered as partially ordered $(\Phi, \Omega, \subseteq$ ). We denote by $\mathrm{R}\{\Omega\}$ (respectively, $\mathrm{R}\{\Omega, \subseteq\}$ ) the class of all algebras (partially ordered algebras) isomorphic to the ones whose elements are binary relations and whose operations are members of $\Omega$. Let $\operatorname{V}\{\Omega\}(\mathrm{V}\{\Omega, \subseteq\})$ be the variety and let $\mathrm{Q}\{\Omega\}(\mathrm{Q}\{\Omega, \subseteq\})$ be the quasi-variety generated by $\mathrm{R}\{\Omega\}$ (generated by $\mathrm{R}\{\Omega, \subseteq\}$ in the class of all partially ordered algebras of the corresponding type).

The following problems naturally arise when the class $\mathrm{R}\{\Omega\}(\mathrm{R}\{\Omega, \subseteq\})$ is considered.

1. Find a system of axioms for the class $\mathrm{R}\{\Omega\}(\mathrm{R}\{\Omega, \subseteq\})$.

2. Find a basis of quasi-identities for the quasi-variety $\mathrm{Q}\{\Omega\}(\mathrm{Q}\{\Omega, \subseteq\})$.

3. Find a basis of identities for the variety $\mathrm{V}\{\Omega\}(\mathrm{V}\{\Omega, \subseteq\})$.

4. Does the class $\mathrm{R}\{\Omega\}(\mathrm{R}\{\Omega, \subseteq\})$ form a quasi-variety?

5. Does the quasi-variety $\mathrm{Q}\{\Omega\}(\mathrm{Q}\{\Omega, \subseteq\})$ form a variety?

Numerous studies have been devoted to solving these problems for various classes of algebras of relations. The first mathematician who treated algebras of relations from the point of view of universal algebra was A. Tarski [6]. He considered algebras of relations (Tarski's algebras of relations) with the following operations: Boolean operations $\cup, \cap,{ }^{-}$; operations of relational product $\circ$ and relational inverse ${ }^{-1}$; constant operations $\Delta$ (diagonal relation), $\varnothing$ (empty relation), $\nabla=U \times U$ (universal relation). He showed that the class $R\left\{0,{ }^{-1}, \cup, \cap,{ }^{-}, \Delta, \varnothing, \nabla\right\}$ is not a quasi-variety and the quasi-variety generated by this class forms a variety [7]. R. Lyndon [8] found the infinite base of identities for this variety.

In this paper, we will consider algebras of relations with one associative primitivepositive operation, i. e., semigroup of relations. Note that the operation $\circ, \cap$ of Tarski's algebras of relations are primitive-positive. These operations are determined by the formulas:

$$
\rho \circ \sigma=\{(u, v):(\exists s)(u, s) \in \rho \wedge(s, v) \in \sigma\}, \quad \rho \cap \sigma=\{(u, v):(u, v) \in \rho \wedge(u, v) \in \sigma\} .
$$

It is well known that the class $\mathrm{R}\{0\}$ coincides with the class of all semigroups and the class $R\{\cap\}$ coincides with the class of all semilattices. The class $R\{0, \subseteq\}$ coincides with the class of all partially ordered semigroups.

The associative operations $\triangleright, \triangleleft$ of restrictive multiplication were introduced and investigated in [9]. These operations are defined as follows:

$$
\rho \triangleright \sigma=\{(u, v):(\exists s)(u, s) \in \rho \wedge(u, v) \in \sigma\}, \quad \rho \triangleleft \sigma=\{(u, v):(\exists s)(u, v) \in \rho \wedge(s, v) \in \sigma\} .
$$

The classes $R\{\triangleright\}$ and $R\{\triangleleft\}$ coincide with the following varieties of semigroups given by the identities $x^{2}=x, x y z=y x z$ and $x^{2}=x, x y z=x z y$ respectively. Characterization of the corresponding partially ordered semigroups requires the additional identities $x y \leqslant y$ and $x y \leqslant x$ respectively. 
There are other associative primitive-positive operations on relations [5]. It is interesting to consider problems 1-5 for classes of semigroups of relations with these operations. We concentrate our attention on the following associative binary primitivepositive operations $*$ and $\star$ on $\operatorname{Rel}(U)$ that is defined in the following way:

$$
\begin{aligned}
& \rho * \sigma=\{(u, v):(\exists s, t, w)(u, s) \in \rho \wedge(t, w) \in \sigma\}, \\
& \rho \star \sigma=\{(u, v):(\exists s, t, w)(s, t) \in \rho \wedge(w, v) \in \sigma\} .
\end{aligned}
$$

Since relations $\rho * \sigma$ and $\rho \star \sigma$ are rectangular, we will treat these operations as the operations of left and right rectangular products respectively. Note that the mapping $f(\rho)=\rho^{-1}$ is an antiisomorphism of the partially ordered semigroups of relations $(\operatorname{Rel}(U), *, \subseteq)$ and $(\operatorname{Rel}(U), \star, \subseteq)$. Therefore, it will be sufficient to study only one of these operations.

\section{MAIN RESULTS}

A partially ordered semigroup is an algebraic system $(A, \cdot, \leqslant)$, where $(A, \cdot)$ is a semigroup and $\leqslant$ is a partial order relation on $A$ that is compatible with multiplication, i. e., $x \leqslant y$ implies $x z \leqslant y z$ and $z x \leqslant z y$ for all $x, y, z \in A$.

The main results are formulated in the following theorems and corollaries. These results provide a solution to problems $1-5$ for classes $\mathrm{R}\{*\}$ and $\mathrm{R}\{*, \subseteq\}$. Their proofs are based on the description of quasi-equational theories of algebras of relations with primitive-positive operations [3].

Theorem 1. The quasi-variety $\mathrm{Q}\{*, \subseteq\}$ forms a variety in the class of all partially ordered semigroups. A partially ordered semigroup $(A, \cdot, \leqslant)$ belongs to the quasivariety $\mathrm{Q}\{*, \subseteq\}$ if and only if it satisfies the identities:

$$
\begin{gathered}
x^{2} y=x y, \\
x y^{2}=x y, \\
x y z=x z y, \\
x \leqslant x^{2}, \\
x y \leqslant x^{2} .
\end{gathered}
$$

Corollary 1. The quasi-variety $\mathrm{Q}\{*\}$ forms a variety. A semigroup $(A, \cdot)$ belongs to the quasi-variety $\mathrm{Q}\{*\}$ if and only if it satisfies the identities (1)-(3).

Theorem 2. The class $\mathrm{R}\{*, \subseteq\}$ does not form a quasi-variety. For a partially ordered semigroup $(A, \cdot, \leqslant)$ the following three conditions are equivalent.

1. $(A, \cdot, \leqslant)$ belongs to the class $\mathrm{R}\{*, \subseteq\}$.

2. One of the following conditions holds:

a) $(A, \cdot, \leqslant)$ satisfies identity (4) and the identity

$$
x y=x^{2}
$$

b) $(A, \cdot, \leqslant)$ contains the zero element o, satisfies identity (4) and the axioms:

$$
\begin{gathered}
y \neq o \Rightarrow x y=x^{2}, \\
o \leqslant x .
\end{gathered}
$$


3. $(A, \cdot \leqslant)$ satisfies identity (4) and the axioms:

$$
\begin{gathered}
x y=x^{2} \vee y z=z y=y, \\
x y=y x=x \Rightarrow x \leqslant z .
\end{gathered}
$$

Corollary 2. The class $\mathrm{R}\{*\}$ does not form a quasi-variety. For a semigroup $(A, \cdot)$ the following three conditions are equivalent.

1. $(A, \cdot)$ belongs to the class $\mathrm{R}\{*\}$.

2. One of the following conditions holds:

a) $(A, \cdot)$ satisfies identity (6);

b) $(A, \cdot)$ contains the zero element and satisfies axiom (7).

3. $(A, \cdot)$ satisfies axiom $(9)$.

\section{PROOFS OF RESULTS}

Step 1. First of all, let us prove the necessity of the conditions of Theorems and Corollaries. Let $(\Phi, *, \subseteq)$ be the partially ordered semigroup of relations on $U$ with the operation of left rectangular product and let $\operatorname{pr}_{1} \rho=\{x:(\exists y)(x, y) \in \rho\}$ be the first projection of the relation $\rho \in \Phi$. It is clear that $\rho * \sigma=p r_{1} \rho \times U=\rho * \rho$ if $\sigma \neq \varnothing$, and $\rho * \sigma=\varnothing$ otherwise. It easily follows that that the identities (1)-(5) hold. It also follows that if $\varnothing \notin \Phi$, then $(\Phi, *)$ satisfies identity (6), and if $\varnothing \in \Phi$, then $\varnothing$ is a zero element and axioms (7) and (8) hold.

Conditions 2 and 3 of Theorem 2 and Corollary 2 are equivalent. Indeed, axiom (6) can be represented as $(\neg(\forall w) y w=w y=y) \Rightarrow x y=x^{2}$. It follows that this axiom is equivalent to identity (6) if $A$ does not contain a zero element, and it is equivalent to axiom (7) otherwise. Axiom (10) just expresses axiom (8) as a universal formula of the first-order language.

Step 2. The proof of sufficiency of conditions of Theorem 1 is based on the result of [3]. Let us give some definitions and notations to formulate this result.

For any formula $\varphi\left(z_{0}, z_{1}, r_{1}, \ldots, r_{m}\right)$ of the first-order predicate calculus with the set of free variables included in $\left\{z_{0}, z_{1}\right\}$ and having $m$ binary predicate symbols $r_{1}, \ldots, r_{m}$, we can associate an $m$-ary operation $F_{\varphi}$ on $\operatorname{Rel}(U)$ defined in the following way:

$$
F_{\varphi}\left(\rho_{1}, \ldots, \rho_{m}\right)=\left\{(u, v) \in U \times U: \varphi\left(u, v, \rho_{1}, \ldots, \rho_{m}\right)\right\}
$$

where $\varphi\left(u, v, \rho_{1}, \ldots, \rho_{m}\right)$ means that the formula $\varphi$ holds whenever $z_{0}, z_{1}$ are interpreted as $u, v$, and $r_{1}, \ldots, r_{m}$ are interpreted as relations $\rho_{1}, \ldots, \rho_{m}$ from $\operatorname{Rel}(U)$. As we stated earlier an operation on relations is called primitive-positive if it can be defined by a first-order formula containing only existential quantifiers and conjunctions in its prenex normal form. Let us describe primitive-positive operations by using graphs [2].

Let $\mathbb{N}$ be the set of all natural numbers and $\mathbb{N}^{0}=\mathbb{N} \cup\{0\}$. A labeled graph is a pair $G=(V(G), E(G))$, where $V(G)$ is a finite set (called a vertex set) and $E(G) \subseteq V(G) \times \mathbb{N} \times V(G)$ is a ternary relation. A triple $(u, k, v) \in E(G)$ is called an edge from $u$ to $v$ labeled by $k$, and it will be graphically represented by $u \cdot \stackrel{k}{\rightarrow} \cdot v$. An input-output-pointed labeled graph is a structure $G=(V(G), E(G), \operatorname{in}(G)$, out $(G))$, where $(V(G), E(G))$ is a labeled graph, $\operatorname{in}(G)$ and out $(G)$ are two distinguished vertices called input and output vertices respectively. The input-output-pointed labeled graph $G$ with $\operatorname{in}(G)=i$ and $\operatorname{out}(G)=o$ is also denoted by $G^{i, o}$. So, we will just call them graphs as it does not lead to confusion. The concept of graph isomorphism is defined 
in a natural way. All graphs will be considered up to isomorphism. Moreover, we will identify graphs differing only in the number of isolated vertices that are distinct from in and out. These isolated vertices will be omitted.

For a given $u \in V(G)$, the number of edges of the form $(u, k, v)$ [respectively, $(v, k, u)$ ] we denote by $\operatorname{deg}^{+}(u)$ [respectively, $\operatorname{deg}^{-}(u)$ ]. Given two input-outputpointed labeled graphs $G_{1}=\left(V_{1}, E_{1} \text {, in } 1 \text {, out }\right)_{1}$ and $G_{2}=\left(V_{2}, E_{2}\right.$, in 2 , out 2$)$, a mapping $f: V_{2} \rightarrow V_{1}$ is called a homomorphism from $G_{2}$ to $G_{1}$ if $f\left(\mathrm{in}_{2}\right)=\mathrm{in}_{1}, f\left(\right.$ out $\left._{2}\right)=$ out $_{1}$, and $(f(u), k, f(v)) \in E_{1}$ whenever $(u, k, v) \in E_{2}$. We write $G_{1} \prec G_{2}$ if there exists a homomorphism from $G_{2}$ to $G_{1}$.

Let $F=F_{\varphi}$ be a primitive positive operation determined by a formula $\varphi$. Then the input-output-pointed labeled graph $G=G_{F}=G_{\varphi}$ associated with $F$ is defined as follows (see [2]). Let $\{0,1, \ldots, n\}$ be the set of all subscripts of individual variables of $\varphi$. We put $G=\left(V, E\right.$, in, out), where $V=\left\{v_{0}, v_{1}, \ldots, v_{n}\right\}$; in $=v_{0}$, out $=v_{1} ; \quad(i, k, j) \in E$ if and only if the atomic formula $r_{k}\left(z_{i}, z_{j}\right)$ occurs in $\varphi$.

Note that the graph $G_{*}=(V, E$, in, out $)$ corresponding to the considered operation * of rectangular product can be described in the following way:

$$
\begin{gathered}
V=\left\{v_{0}, v_{1}, v_{2}, v_{3}, v_{4}\right\}, \quad E=\left\{\left(v_{0}, 1, v_{3}\right),\left(v_{2}, 2, v_{4}\right)\right\}, \quad \text { in }=v_{0}, \quad \text { out }=v_{1}, \\
\text { in }=v_{0} \cdot \stackrel{1}{\longrightarrow} \cdot v_{3} \quad v_{2} \cdot \stackrel{2}{\longrightarrow} \cdot v_{4} \quad \cdot v_{1}=\text { out. }
\end{gathered}
$$

Let $G=(V, E$, in, out $)$ and $G_{k}=\left(V_{k}, E_{k}\right.$, in $_{k}$, out $\left._{k}\right)(k=1,2, \ldots, n)$ be graphs with pairwise disjoint vertex sets. The composition $G\left(G_{1}, G_{2}, \ldots, G_{n}\right)$ is the graph constructed as follows [3]: take $G$ and substitute every edge $(u, k, v) \in E$ by the graph $G_{k}$ identifying the input vertex $\operatorname{in}_{k}$ with $u$ and the output vertex out $k$ with $v$.

Let us consider the set $\Lambda=\left\{x_{1}, \ldots, x_{n}, \ldots\right\}$ of individual variables that are interpreted as elements of a semigroup. A semigroup term $p$ is a word in the alphabet $\Lambda$, i. e., an expression of the form $x_{j_{1}} x_{j_{2}} \ldots x_{j_{m-1}} x_{j_{m}}$. For convenience, we will also use other letters of the Latin alphabet as variables.

Suppose that $p=x_{j_{1}} x_{j_{2}} \ldots x_{j_{m-1}} x_{j_{m}}$ be the term of a semigroup that satisfies identities (1)-(3). Then without loss of generality, we can assume that all variables $x_{j_{1}}, \ldots, x_{j_{m}}$ are different. Moreover, we can also presume that variables $x_{j_{2}}, \ldots, x_{j_{m}}$ can be arbitrarily permuted. In what follows, we will use these properties without special mentions.

For any semigroup term $p$ we define the graph $G(p)=(V(p), E(p), \operatorname{in}(p)$, out $(p))$ in the following inductive way:

1) if $p=x_{k}$, then $G(p)$ is the following graph: in $\stackrel{k}{\rightarrow} \cdot$ out;

2) if $p=p_{1} p_{2}$, then $G(p)=G_{*}\left(G\left(p_{1}\right), G\left(p_{2}\right)\right)$.

For any term $p=x_{j_{1}} x_{j_{2}} \ldots x_{j_{m-1}} x_{j_{m}}$ the graph $G(p)$ has the following form:

$$
\text { in }=v_{0} \cdot \stackrel{j_{1}}{\longrightarrow} \cdot \stackrel{j_{2}}{\longrightarrow} \cdot \cdots \cdot \stackrel{j_{m-1}}{\longrightarrow} \cdot \stackrel{j_{m}}{\longrightarrow} \cdot \cdot v_{1}=\text { out. }
$$

Let $G$ be a labeled graph, $u, v \in V(G)=\left\{v_{0}, v_{1}, \ldots, v_{n}\right\}$, and $Q$ be an input-output-pointed labeled graph. Without loss of generality, we can suppose that $V(Q)=$ $=\left\{w_{0}, w_{1}, \ldots, w_{m}\right\}, \operatorname{in}(Q)=w_{0}=u, \operatorname{out}(Q)=w_{1}=v$, and $V(G) \cap V(Q)=\{u, v\}$. The labeled grath $(V(G) \cup V(Q), E(G) \cup E(Q))$ we denote by $G[u, v, Q]$. Note that the edges set of $G[u, v, Q]$ can be represented as $\left\{v_{0}, v_{1}, \ldots, v_{n}, v_{n+1}, \ldots, v_{n+m-1}\right\}$, where $v_{n+1}=w_{2}, \ldots, v_{n+m-1}=w_{m}$. Virtually, the graph $G[u, v, Q]$ is obtained from the graph $G$ by "gluing" the graph $Q$ to the vertices $u$ and $v$. 
Define an $n$-system to be a pair $\omega=(\alpha, \beta)$, where $\alpha, \beta:\{1, \ldots, n\} \rightarrow \mathbb{N}^{0}$ are mappings, $\alpha(k), \beta(k)<2+(k-1)(m-2)$ for all $k=1, \ldots, n$, and $m$ is the number of vertices of the graph that determines the considered operation on relations (for the operation $*$ we have $m=5$ and $\alpha(k), \beta(k)<3 k-1)$.

Given an $n$-system $\omega=(\alpha, \beta)$, we construct by induction the sequence of graphs $G_{0} \subseteq \cdots \subseteq G_{n}=G_{\omega}$. We put $G_{0}: v_{0} \cdot \stackrel{1}{\rightarrow} \cdot v_{1}$, and for $k=1, \ldots, n$ we put:

$$
G_{k}=G_{k-1}\left[v_{\alpha(k)}, v_{\beta(k)}, G\left(x_{2 k} x_{2 k+1}\right)\right] .
$$

The following proposition presents the result of [3] formulated for the class $\mathrm{R}\{*, \subseteq\}$.

Proposition 1. A partially ordered semigroup $(A, \cdot, \leqslant)$ belongs to the quasi-variety $\mathrm{Q}\{*, \subseteq\}$ if and only if it satisfies the quasi-identity

$$
\left(\bigwedge_{k=1}^{n} p_{k} \leqslant x_{2 k} x_{2 k+1}\right) \Rightarrow x_{1} \leqslant p_{0}
$$

for every $n$-system $\omega=(\alpha, \beta)$ and arbitrary terms $p_{0}, \ldots, p_{n}$ such that $G_{\omega}^{v_{0}, v_{1}} \prec G\left(p_{0}\right)$ and $G_{k-1}^{v_{\alpha(k)}, v_{\beta(k)}} \prec G\left(p_{k}\right)$.

Step 3. We are ready to prove the sufficiency of the conditions of Theorem 1. Let $\omega=(\alpha, \beta)$ be the $n$-system and $p_{0}, p_{1}, \ldots, p_{n}$ be the terms such that $G_{\omega}^{v_{0}, v_{1}} \prec G\left(p_{0}\right)$ and $G_{k-1}^{v_{\alpha(k)}, v_{\beta(k)}} \prec G\left(p_{k}\right)$ for $k=1, \ldots n$. This system corresponds to the sequence of graphs $G_{0} \subseteq G_{1} \subseteq \cdots \subseteq G_{n}=G_{\omega}$, where $G_{k}=\left(V_{k}, E_{k}\right)$ for $k=0, \ldots, n$. For any $k \leqslant n$ we have that $V_{k}=\left\{v_{0}, v_{1}, \ldots, v_{3 k-1}, v_{3 k}, v_{3 k+1}\right\}$ and

$$
E_{k}=\left\{\left(v_{0}, 1, v_{1}\right)\right\} \cup\left\{\left(v_{\alpha(i)}, 2 i, v_{3 i-1}\right),\left(v_{3 i}, 2 i+1, v_{v_{3 i+1}}\right): i=1, \ldots, k\right\} .
$$

Let us prove by induction on $k$ that $\alpha(k)=3 j$ for some $j=0, \ldots, k$; deg $v_{3 i}=0$, $d e g^{+} v_{3 i}>0$ for any $i=0, \ldots, k$, and $d e g^{-} v=1, d e g^{+} v=0$ for all other vertices $v$ of the graph $G_{k}$.

Let $k=1$. Since $G_{0}^{v_{\alpha(1)}, v_{\beta(1)}} \prec G\left(p_{1}\right)$, we have $p_{1}=x_{1}$ or $p_{1}=x_{1}^{2}$, and $\alpha(1)=0$, $\beta(1)=1 ; \operatorname{deg}^{-} v_{0}=0, \operatorname{deg}^{+} v_{0}=2 ; \operatorname{deg}^{-} v_{1}=1, \operatorname{deg}^{+} v_{1}=0 ; \operatorname{deg}^{-} v_{2}=1, \operatorname{deg}^{+} v_{2}=0$; $\operatorname{deg}^{-} v_{3}=0, \operatorname{deg}^{+} v_{3}=1 ; \operatorname{deg}^{-} v_{1}=1$, $\operatorname{deg}^{+} v_{4}=0$.

Suppose now that it holds for $k-1$, and let us show that this is true for $k$. Since $G_{k-1}^{v_{\alpha(k)}, v_{\beta(k)}} \prec G\left(p_{k}\right)$, according to the definition of a graph homomorphism, we get $d e g^{+} v_{\alpha(k)}>0$. Then according to the induction assumption we get $\alpha(k)=3 j$ for some $j=0, \ldots, k ; \operatorname{deg}^{-} v_{3 i}=0, \operatorname{deg}^{+} v_{3 i}>0$ for any $i=0, \ldots, k$, and $\operatorname{deg}^{-} v=1, \operatorname{deg}^{+} v=0$ for all other vertices $v$ of the graph $G_{k}$.

Let $(A, \cdot, \leqslant)$ be a partially ordered semigroup satisfying identities (1)-(5). Suppose that the premise of quasi-identity (11) holds for some values of the variables $x_{1}=a_{1}$, $x_{2}=a_{2}, x_{3}=a_{3}, \ldots, x_{2 n}=a_{2 n}, x_{2 n+1}=a_{2 n+1}$, i. e., $p_{k}(\vec{a}) \leqslant a_{2 k} a_{2 k+1}$ for all $k=1, \ldots, n$, where $\vec{a}=\left(a_{1}, a_{2}, \ldots, a_{2 n+1}\right)$. Let $p_{0}=x_{j_{1}} x_{j_{2}} \ldots x_{j_{m-1}} x_{j_{m}}$. Note that $G_{k}^{v_{0}, v_{1}} \prec G\left(p_{0}\right)$ if and only if $\left\{x_{j_{1}}, x_{j_{2}}, \ldots, x_{j_{m-1}}, x_{j_{m}}\right\} \subseteq\left\{x_{1}, x_{2}, \ldots, x_{2 k}, x_{2 k+1}\right\}, x_{j_{1}}=x_{1}$ or $x_{j_{1}}=x_{3 i}$ for some $0<i \leqslant k$ such that $\alpha(i)=0$.

Let $\max \left(p_{0}\right)$ be the greatest $k$ such that at least one of the variables $x_{2 k}$ or $x_{2 k+1}$ is included in the term $p_{0}$. Let us prove by induction on $\max \left(p_{0}\right)$ that $a_{1} \leqslant p_{0}(\vec{a})$. If $\max \left(p_{0}\right)=0$, then $p=x_{1}$ or $p_{0}=x_{1}^{2}$. Thus, using identity (4) we obtain $a_{1} \leqslant p_{0}(\vec{a})$. 
Suppose now that $a_{1} \leqslant p_{0}(\vec{a})$ holds for $\max \left(p_{0}\right)=k-1$, and let us show that this is true for $\max \left(p_{0}\right)=k$.

If both variables $x_{2 k}, x_{2 k+1}$ are included in $p_{0}$, then the following cases are possible:

1) $p_{0}=x_{2 k} x_{2 k+1} x_{j_{3}} \ldots x_{j_{m-1}} x_{j_{m}}$, then $\alpha(k)=0$. It follows that $G_{k}^{v_{0}, v_{1}} \prec G\left(p_{k} x_{j_{3}} \ldots\right.$ $\left.x_{j_{m}}\right)$, and by the induction assumption we get

$$
a_{0} \leqslant p_{k}(\vec{a}) a_{j_{3}} \ldots a_{j_{m-1}} a_{j_{m}} \leqslant a_{2 k} a_{2 k+1} a_{j_{3}} \ldots a_{j_{m-1}} a_{j_{m}}=p_{0}(\vec{a})
$$

2) $p_{0}=x_{j_{1}} x_{2 k} x_{2 k+1} x_{j_{4}} \ldots x_{j_{m-1}} x_{j_{m}}$, then using the induction assumption we get

$$
a_{0} \leqslant a_{j_{1}} p_{k}(\vec{a}) a_{j_{4}} \ldots a_{j_{m-1}} a_{j_{m}} \leqslant a_{j_{1}} a_{2 k} a_{2 k+1} a_{j_{4}} \ldots a_{j_{m-1}} a_{j_{m}}=p_{0}(\vec{a}) .
$$

If only one of the variables $x_{2 k}$ or $x_{2 k+1}$ is included in $p_{0}$, then the following cases are possible:

3) $p_{0}=x_{2 k} x_{j_{2}} \ldots x_{j_{m-1}} x_{j_{m}}$, then $\alpha(k)=0$. It follows that $G_{k}^{v_{0}, v_{1}} \prec G\left(p_{k} x_{j_{2}} \ldots x_{j_{m}}\right)$, and by the induction assumption we get

$$
\begin{aligned}
& a_{0} \leqslant p_{k}(\vec{a}) a_{j_{2}} \ldots a_{j_{m-1}} a_{j_{m}} \leqslant a_{2 k} a_{2 k+1} a_{j_{3}} \ldots a_{j_{m-1}} a_{j_{m}} \leqslant \\
& \stackrel{(5)}{\leqslant} a_{2 k}^{2} a_{j_{2}} \ldots a_{j_{m-1}} a_{j_{m}} \stackrel{\stackrel{(1)}{=}}{=} a_{2 k} a_{j_{2}} \ldots a_{j_{m-1}} a_{j_{m}}=p_{0}(\vec{a}) ;
\end{aligned}
$$

4) $p_{0}=x_{j_{1}} x_{2 k} x_{j_{3}} \ldots x_{j_{m-1}} x_{j_{m}}$, then using the induction assumption we get

$$
\begin{aligned}
& a_{0} \leqslant a_{j_{1}} p_{k}(\vec{a}) a_{j_{3}} \ldots a_{j_{m-1}} a_{j_{m}} \leqslant a_{j_{1}} a_{2 k} a_{2 k+1} a_{j_{3}} \ldots a_{j_{m-1}} a_{j_{m}} \leqslant \\
& \stackrel{(5)}{\leqslant} a_{j_{1}} a_{2 k}^{2} a_{j_{3}} \ldots a_{j_{m-1}} a_{j_{m}} \stackrel{(2)}{=} a_{j_{1}} a_{2 k} a_{j_{3}} \ldots a_{j_{m-1}} a_{j_{m}}=p_{0}(\vec{a}) ;
\end{aligned}
$$

5) $p_{0}=x_{j_{1}} x_{2 k+1} x_{j_{3}} \ldots x_{j_{m-1}} x_{j_{m}}$, then using the induction assumption we get

$$
\begin{aligned}
& a_{0} \leqslant a_{j_{1}} p_{k}(\vec{a}) a_{j_{4}} \ldots a_{j_{m-1}} a_{j_{m}} \leqslant a_{j_{1}} a_{2 k} a_{2 k+1} a_{j_{4}} \ldots a_{j_{m-1}} a_{j_{m}} \leqslant \\
& \stackrel{(5)}{\leqslant} a_{j_{1}} a_{2 k+1}^{2} a_{j_{4}} \ldots a_{j_{m-1}} a_{j_{m}} \stackrel{(2)}{=} a_{j_{1}} a_{2 k+1} a_{j_{4}} \ldots a_{j_{m-1}} a_{j_{m}}=p_{0}(\vec{a}) .
\end{aligned}
$$

Thus, we have proved that the partially ordered semigroup $(A, \cdot, \leqslant)$ satisfies quasiidentity (11). Therefore, according to Proposition 1 we have $(A, \cdot, \leqslant) \in \mathrm{Q}\{*, \subseteq\}$. This completes the proof of Theorem 1.

Step 4. Let us prove Corollary 1. Suppose that a semigroup $(A, \cdot)$ satisfies identities (1)-(3) and $A^{2}=\left\{a^{2}: a \in A\right\}$. We define the relation $\leqslant$ on the set $A$ by setting

$$
\leqslant=\left\{(x, y) \in A \times A^{2}: x^{2}=y x\right\} \cup\{(x, x) \in A \times A: x \in A\} .
$$

Let us show that $(A, \cdot, \leqslant)$ is the partially ordered semigroup satisfying identities (4) and $(5)$. The reflexivity of the relation $\leqslant$ follows directly from the definition.

To prove the transitivity assume that $x \leqslant y$ and $y \leqslant z$. Without loss of generality, we can suppose that $x \neq y$ and $y \neq z$. Then $x^{2}=y x, y^{2}=z y$ and $y^{2}=y, z^{2}=z$, hence $x^{2}=y x=z y x=z x^{2} \stackrel{(2)}{=} z x$, i. e., $x \leqslant z$. Thus, $\leqslant$ is transitive.

Assume that $x \leqslant y, y \leqslant x$ and $x \neq y$. Then $x^{2}=y x, y^{2}=x y$ and $x^{2}=x, y^{2}=y$, hence $x=x^{2}=y x \stackrel{(1)}{=} y^{2} x=x y x \stackrel{(3)}{=} x^{2} y \stackrel{(1)}{=} x y=y^{2}=y$. This contradicts the assumption $x \neq y$. Thus, $\leqslant$ is a partially order relation. 
Let us show that the relation $\leqslant$ is compatible with multiplication. Suppose that $x \leqslant y$ and $x \neq y$. Then $x^{2}=y x$ and $y^{2}=y$, hence $(x z)^{2}=x z x z \stackrel{(3)}{=} x^{2} z^{2}=y x z^{2} \stackrel{(3)}{=}(y z)(x z)$ and $(y z)^{2}=y z y z \stackrel{(3)}{=} y^{2} z^{2} \stackrel{(1)}{=} y z^{2} \stackrel{(2)}{=} y z$. Thus, $x z \leqslant y z$. Further, $(z x)^{2}=z x z x \stackrel{(3)}{=} z^{2} x^{2}=$ $=z^{2} y x \stackrel{(3)}{=}(z y)(z x)$ and $(z y)^{2}=z y z y \stackrel{(3)}{=} z^{2} y^{2} \stackrel{(1)}{=} z y^{2} \stackrel{(2)}{=} z y$. Thus, $z x \leqslant z y$.

Since $x^{2} \stackrel{(3)}{=} x^{3}=x^{2} x$ and $\left(x^{2}\right)^{2}=x^{4} \stackrel{(1)}{=} x^{2}$, we have $x \leqslant x^{2}$. Since $(x y)^{2}=$ $=x y x y \stackrel{(3)}{=} x x y^{2} \stackrel{(1)}{=} x^{2} x y^{2} \stackrel{(2)}{=} x^{2} x y$ and $\left(x^{2}\right)^{2}=x^{4} \stackrel{(1)}{=} x^{2}$, we have $x y \leqslant x^{2}$. Therefore, $(A, \cdot, \leqslant)$ satisfies identities (1) and $(2)$, hence $(A, \cdot, \leqslant) \in \mathrm{Q}\{*, \subseteq\}$ and $(A, \cdot) \in \mathrm{Q}\{*\}$. This completes the proof of Corollary 1.

Step 5. Let us prove Theorem 2 and Corollary 2. First of all, it is easy to see that for $U \neq \varnothing$, the Cartesian square of the semigroup $(\operatorname{Rel}(U), *)$ of relations contains the zero element $(\varnothing, \varnothing)$ and does not satisfy axiom $(7)$. It follows that the classes $\mathrm{R}\{*\}$ and $\mathrm{R}\{*, \subseteq\}$ do not form quasi-varieties.

Lemma 1. Let $\left\{U_{j}: j \in J\right\}$ be a family of pairwise non-intersecting sets and $U=\bigcup\left\{U_{j}: j \in J\right\}$. If a partially ordered semigroup $(A, \cdot, \leqslant)$ is a subdirect product of a family $\left\{\left(\Phi_{j}, *, \subseteq\right): j \in J\right\}$ of partially ordered semigroups of relations on $U_{j}$ and satisfies identity (6), then $(A, \cdot, \leqslant)$ isomorphically embedded in $(\operatorname{Rel}(U), *, \subseteq)$.

Proof. Let $\varphi_{j}: A \longrightarrow \Phi_{j}$ be the corresponding surjective homomorphisms from $A$ on the components of the direct product $\prod\left\{\Phi_{j}: j \in J\right\}$. According to the properties of homomorphic images, we have that all components $\left(\Phi_{j}, *, \subseteq\right)$ satisfy identity (6). Hence, for all $j \in J$ we have $\varnothing \notin \Phi_{j}$ or $\Phi_{j}=\{\varnothing\}$. It follows that $(A, \cdot, \leqslant)$ is a subdirect product of the family $\left\{\left(\Phi_{j}, *, \subseteq\right): j \in J_{0}\right\}$, where $J_{0}=\left\{j \in J: \varnothing \notin \Phi_{j}\right\}$.

For a given $a \in A$, we put $\rho_{j}^{a}=\varphi_{j}(a)$. We define a mapping $\varphi: A \longrightarrow \operatorname{Rel}(U)$ in the following way. We put $\varphi(a)=\bigcup\left\{p r_{1} \rho_{j}^{a}: j \in J_{0}\right\} \times U$, if $a^{2}=a$, and $\varphi(a)=\bigcup\left\{\rho_{j}^{a}: j \in J_{0}\right\} \cup \bigcup\left\{\varphi(b): b^{2}=b \leqslant a\right\}$ otherwise. Let us show that $\varphi$ is an isomorphic embedding $(A, \cdot, \leqslant)$ in $(\operatorname{Rel}(U), *, \subseteq)$.

Note that $\varphi(a) \cap U_{j} \times U_{j}=\rho_{j}^{a}$ for all $a \in A$. It follows that $\varphi(a) \subseteq \varphi(a)$ if and only if $a \leqslant b$. Furthermore, since $\varnothing \notin \Phi_{j}$ and $(a b)^{2}=a b a b \stackrel{(3)}{=} a a b b \stackrel{(2)}{=} a a b \stackrel{(1)}{=} a b$, we have

$$
\begin{gathered}
\varphi(a b)=\bigcup\left\{p r_{1} \rho_{j}^{a b}: j \in J_{0}\right\} \times U=\left(\bigcup\left\{p r_{1} \rho_{j}^{a}: j \in J_{0}\right\} \times U=\right. \\
=p r_{1} \varphi(a) \times U=\varphi(a) * \varphi(b) .
\end{gathered}
$$

Lemma 2. Suppose that $(A, \cdot, \leqslant)$ satisfies identities (4) and (6). Then $(A, \cdot, \leqslant)$ belongs to $\mathrm{R}\{*, \subseteq\}$.

Proof. It is easy to see that identity (6) imlies identities (1)-(3) and (5). Hence, if $(A, \cdot \leqslant)$ satisfies identities (4) and (6), then according to Theorem 1 we have that $(A, \cdot, \leqslant) \in \mathrm{Q}\{*, \subseteq\}$. In respect that the class $\mathrm{R}\{*, \subseteq\}$ is axiomatizable [1], we obtain that $(A, \cdot, \leqslant)$ is a subdirect product of a family of partially ordered semigroups from $\mathrm{R}\{*, \subseteq\}$. Hence, according to Lemma 1 , we obtain that $(A, \cdot, \leqslant)$ belongs to $\mathrm{R}\{*, \subseteq\}$.

Lemma 3. Suppose that $(A, \cdot)$ satisfies identity (6). Then $(A, \cdot)$ belongs to $\mathrm{R}\{*\}$.

Proof. If $(A, \cdot)$ satisfies identity (6), then it satisfies identities (1)-(3). Let $\leqslant$ be the partial order relation constructed in the proof of Corollary 1. Then by Lemma 3 we have $(A, \cdot, \leqslant) \in \mathrm{R}\{*, \subseteq\}$. Therefore, $(A, \cdot) \in \mathrm{R}\{*\}$. 
Lemma 4. Suppose that $(A, \cdot)$ contains the zero element $o$ and satisfies axiom (7). Then $(A, \cdot)$ satisfies identity (6) or $a b \neq o$ for all $a, b \neq o$.

Proof. If there exist elements $a \neq o$ and $b \neq o$ such that $a b=o$, then for all $x, y \neq o$ we have $x a \stackrel{(7)}{=} x^{2}, y b \stackrel{(7)}{=} y^{2}$, and $x a b=o$, hence $x y \stackrel{(1)}{=} x^{2} y \stackrel{(2)}{=} x^{2} y^{2}=x a y b \stackrel{(3)}{=} x y a b=$ $=x y_{0}=o$. It follows that $x y=x^{2}$ for all $x, y, z \in A$, i. e., $(A, \cdot)$ satisfies identity (6).

Suppose that $(A, \cdot, \leqslant)$ contains the zero element $o$ and satisfies identity (4) and axioms (7) and (8). We put $B=A \backslash\{o\}$. According to Lemmas 3 and 4 we can suppose that $x y \in B$ for all $x, y \in B$, and $(B, \cdot, \leqslant)$ satisfies identities $(4)$ and $(6)$, hence $(B, \cdot, \leqslant)$ belongs to $\mathrm{R}\{*, \subseteq\}$. It means that there exists an isomorphism $F$ from the partially ordered semigroup $(B, \cdot, \leqslant)$ to some partially ordered semigroup of relations $(\Phi, *, \subseteq)$ and $\varnothing \notin \Phi$. Putting $F(o)=\varnothing$, we get the isomorphism from $(A, \cdot, \leqslant)$ to $(\Phi \cup\{\varnothing\}, *, \subseteq)$. Therefore, $(A, \cdot, \leqslant)$ belongs to $\mathrm{R}\{*, \subseteq\}$. This completes the proof of Theorem 2 .

Suppose that $(A, \cdot)$ contains the zero element $o$ and satisfies axiom (7), $B=A \backslash\{o\}$, and let $\leqslant$ be the partial order relation on $B$ constructed in the proof of Theorem 2 . Extend the relation $\leqslant$ on $A$ by putting $o \leqslant a$ for all $a \in A$. Then $(A, \cdot, \leqslant)$ satisfies the conditions of Theorem 2 , hence $(A, \cdot, \leqslant) \in \mathrm{R}\{*, \subseteq\}$. Therefore, $(A, \cdot)$ belongs to $\mathrm{R}\{*\}$. This completes the proof of Corollary 2.

\section{CONCLUSION}

The results of the article show that the classes $\mathrm{R}\{*\}, \mathrm{R}\{*, \subseteq\}, \mathrm{R}\{\star\}, \mathrm{R}\{\star, \subseteq\}$ are finitely axiomatizable and are not quasi-varieties; the quasivarieties generated by these classes are finitely based varieties, and also that $\mathrm{R}\{\triangleleft\} \subset \mathrm{V}\{*\}, \mathrm{R}\{\triangleleft, \subseteq\} \subset \mathrm{V}\{*, \subseteq\}$, $\mathrm{R}\{\triangleright\} \subset \mathrm{V}\{\star\}, \mathrm{R}\{\triangleright, \subseteq\} \subset \mathrm{V}\{\star, \subseteq\}$.

\section{References}

1. Schein B. M. Relation algebras and function semigroups. Semigroup Forum, 1970, vol. 1, pp. $1-62$.

2. Böner P., Pöschel F. R. Clones of operations on binary relations. Contributions to general algebra, 1991, vol. 7, pp. 50-70.

3. Bredikhin D. A. On quasi-identities of algebras of relations with Diophantine operations. Sib. Math. J., 1997, vol. 38, pp. 23-33. DOI: https://doi.org/10.1007/BF02674896

4. Bredikhin D. A. On algebras of relations with Diophantine operations. Dokl. Math., 1998, vol. 57, no. 3, pp. 435-436.

5. Bredikhin D. A. On relation algebras with general superpositions. Algebraic Logic, 1998, vol. 54, pp. 111-124.

6. Tarski A. On the calculus of relations. J. Symbolic Logic, 1941, vol. 6, pp. 73-89.

7. Tarski A. Contributions to the theory of models, III. Proc. Konikl. Nederl. Akad. Wet., 1956, vol. 58, pp. 56-64.

8. Lyndon R. C. The representation of relation algebras, II. Ann. Math., 1956, vol. 63, no. 2, pp. 294-307. DOI: https://doi.org/10.2307/1969611

9. Wagner V. V. Restrictiv semigroups. Izv. Vyssh. Uchebn. Zaved. Mat., 1962, no. 6, pp. 19-27 (in Russian).

\section{Cite this article as:}

Bredikhin D. A. On Semigroups of Relations with the Operation of Left and Right Rectangular Products. Izv. Saratov Univ. (N. S.), Ser. Math. Mech. Inform., 2020, vol. 20, iss. 3, pp. $280-$ 289. DOI: https://doi.org/10.18500/1816-9791-2020-20-3-280-289 
УДК 501.1

\title{
О полугруппах отношений с операцией левого и правого прямоугольного произведения
}

\begin{abstract}
Д. А. Бредихин
Бредихин Дмитрий Александрович, доктор фризико-математических наук, профрессор кафредры математики и моделирования, Саратовский государственный технический университет имени Гагарина Ю. А., Россия, 410054, г. Саратов, ул. Политехническая, д. 77, bredikhin@mail.ru

Множество бинарных отношений, замкнутое относительно некоторой совукупности операций над ними, образует алгебру, называемую алгеброй отношений. Класс всех алгебр (частично упорядоченных алгебр), изоморфнных алгебрам (частично упорядоченным теоретико-множественным включением $\subseteq$ алгебрам) отношений с операциями из $\Omega$, обозначим $\mathrm{R}\{\Omega\}(R\{\Omega, \subseteq\})$. Операция над бинарными отношениями называется примитивно-позитивной, если она может быть определена фрормулой, содержащей в своей префрексной нормальной фрорме лишь кванторы существования и операцию конъюнкции. В работе рассматриваются алгебры отношений с ассоциативными примитивно-позитивными операциями $* и \star$, определяемыми следующими фоормулами: $\rho * \sigma=\{(u, v):(\exists s, t, w)(u, s) \in \rho \wedge(t, w) \in \sigma\}$ и $\rho \star \sigma=\{(u, v):(\exists s, t, w)(s, t) \in \rho \wedge(w, v) \in \sigma\}$ соответственно. Найдены системы аксиом для классов $\mathrm{R}\{*\}, \mathrm{R}\{*, \subseteq\}, \mathrm{R}\{\star\}, \mathrm{R}\{\star, \subseteq\}$ и базисы тождеств для порожденных этими классами квазимногообразий и многообразий.

Ключевые слова: алгебра отношений, примитивно-позитивная операция, тождество, многообразие, квазитождество, квазимногообразие, полугруппа, частично упорядоченная полугруппа.
\end{abstract}

Поступила в редакцию: 11.06.2019 / Принята: 28.06.2019 / Опубликована: 31.08.2020

Статья опубликована на условиях лицензии Creative Commons Attribution License (CC-BY 4.0)

Образец для цитирования:

Bredikhin D. A. On Semigroups of Relations with the Operation of Left and Right Rectangular Products [Бредихин Д. А. О полугруппах отношений с операцией левого и правого прямоугольного произведения] // Изв. Сарат. ун-та. Нов. сер. Сер. Математика. Механика. Информатика. 2020. Т. 20, вып. 3. С. 280-289. DOI: https://doi.org/10.18500/1816-97912020-20-3-280-289 